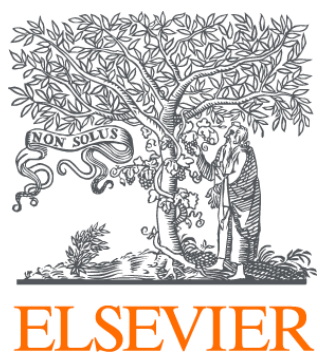

Since January 2020 Elsevier has created a COVID-19 resource centre with free information in English and Mandarin on the novel coronavirus COVID-

19. The COVID-19 resource centre is hosted on Elsevier Connect, the company's public news and information website.

Elsevier hereby grants permission to make all its COVID-19-related research that is available on the COVID-19 resource centre - including this research content - immediately available in PubMed Central and other publicly funded repositories, such as the WHO COVID database with rights for unrestricted research re-use and analyses in any form or by any means with acknowledgement of the original source. These permissions are granted for free by Elsevier for as long as the COVID-19 resource centre remains active. 


\section{SC159 Increased mortality among men diagnosed with impaired fertility: analysis of us claims data}

F. Del Giudice, F. Belladelli, G.M. Busetto, E. De Berardinis, A. Sciarra, M. Maggi, M.L. Eisenberg, (Roma)

Author of the Study: To determine whether male infertility or impaired spermatogenesis is associated with mortality.

Materials and Methods: The Optum de-identified Clinformatics Data Mart database was queried from 2003 to 2017. Infertile men were compared to subjects undergoing semen analysis (ie, infertility testing). Infertile men with oligozoospermia or azoospermia were included. Mortality was determined by data linkage to the Social Security Administration Death Master File. Results were adjusted for age, smoking, obesity, year of evaluation, and health care visits as well as for most prevalent comorbidities. We separately examined men with prevalent or incident cardiovascular disease and cancer diagnoses to determine associations with mortality.

Results: A total of 134,796 infertile men and 242,282 controls were followed for a mean of 3.6 and 3.1 years respectively. Overall, infertile men had a higher risk of death (Hazard Ratio [HR] = 1.42, 95\% CI: 1.27 1.60) The diagnosis of azoospermia was associated with a significantly increased risk of death $(\mathrm{HR}=2.01,95 \% \mathrm{CI}: 1.60-2.53)$ with a higher trend among men with oligospermia (HR: $1.17,95 \% \mathrm{CI}: 0.92-1.49$ ) compared to controls. Subanalysis was done excluding prevalent cardiovascular and malignant disease (alone and combined) showing similar hazard ratios.

Conclusions: Male infertility is associated with a higher risk of mortality especially among azoospermic men. Prevalent disease (which is known to be higher among infertile men) did not explain the higher risk of death among infertile men. The implications for treatment and surveillance of infertile men require further study.

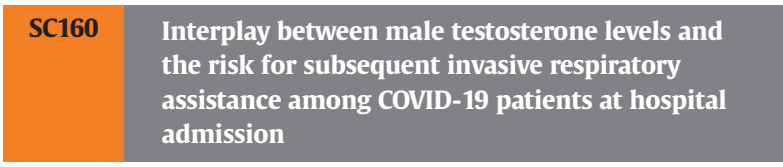

F. Del Giudice, S. Salciccia, V. Gentile, C. Mastroianni, P. Pasculli, G. Di Lascio, M. Moriconi, M.R. Ciardi, M. Maggi, E. De Berardinis, A. Sciarra, (Roma)

Author of the Study: Male sex, and older age were found to be significant determinants for severe SARS-CoV-2 phenotype supporting the hypothesis that hormonal constitution may be an etiology for both COVID- 19 susceptibility and acute respiratory distress syndrome (ARDS) development. Higher serum total testosterone (TT) levels are associated with an immunosuppressive role on different components of the immune cell-mediated response. We aimed to evaluate whether serum TT levels among a cohort of COVID-19 men at the time of hospital admission were associated with the need for "invasive" oxygenation strategy and may allow for patient monitoring and predict disease outcome.

Materials and Methods: This is a single institution study, analyzing a cohort of 29 men diagnosed with COVID-19. Patients' haematochemical and clinical characteristics were recorded at the time of hospital admission. Patients were stratified according to the need of $\mathrm{O} 2$ assistance (i.e., none versus invasive, such as Ventimask, CPAP, intubation). Linear regression was used to examine the correlation between serum TT and haemato-chemical variables of interest. Oneway ANOVA was used to test the differences between continuous TT and IL- 6 values for the different respiratory assistance strategies. Locally weighted scatter-plot smoother function was used to graphically depict the relationship concerning these two variables and the probability of their mutual interaction for the previously defined thresholds.

Results: After adjusting for Age-adjusted Charlson Comorbidity Index, history of hypertension, dyslipidemia, and smoking status, higher serum TT levels $(\mathrm{ng} / \mathrm{ml})$ were found independently associated with a lower odd of invasive oxygenation (odds ratio: $0.43,95 \% \mathrm{CI} 0.23-0.85$; $\mathrm{p}=0.016)$. A significant negative correlation was found between TT and C-reactive protein, $\mathrm{pH}$, Interleukine-6 (IL-6), and D-Dimer. One-way ANOVA analysis confirmed as thresholds of interest $<3.5-4 \mathrm{ng} / \mathrm{ml}$ for impaired T, while identifying $>50 \mathrm{pg} / \mathrm{ml}$ for significantly elevated IL-6. Conclusions: In our cohort, while age was not significantly different among the two groups, TT levels were significantly lower in the ARDS group and associated with worse clinical COVID-19 phenotype. Considering the observed inverse relationship between IL-6 and TT levels, we speculate that greater TT levels could serve as hormonal shield against the COVID- 19-related cytokine syndrome and that low TT levels may allow the viral infection due to a loss of immunosuppressive effect of TT. Although we were able to identify serum TT levels at hospital admission as a potential biomarker for the requirement for invasive respiratory assistance, the interplay between TT levels and COVID-19 require additional study to determine the utility of TT in clinical practice.

\section{SC161 Evaluation of SARS-CoV-2 and semen quality in COVID-19 patients}

F.I. Scroppo, F. Gaeta, E. Illiano, E. Costantini, F. Trama, M.R. Gismondo, S. Brancorsini, R. Bartoletti, A. Zucchi, (Varese)

Author of the Study: The aim of this study was to evaluate the possible presence of SARS-CoV-2 in semen samples collected by young men with COVID-19 and their semen quality looking for a possible relationship between the infectious disease and fertility.

Materials and Methods: In this prospective study we enrolled 15 consecutive men (age 18-50 ys) with positive oropharyngeal swab to SARS-CoV 2 and mild to moderate disease according to NCPPCP (7th edition) classification. Exclusion criteria were the presence of coomorbidities potentially related to infertility.Blood samples for the assay of $\mathrm{FSH}, \mathrm{LH}$, testosterone, procalcitonin, interleukin $6, \mathrm{C}$ reactive protein was obtained. A semen sample was collected to detect SARSCoV viral RNA by the automated Real- Time PCR ELITe InGenius ${ }^{\circledR}$ system and the GeneFinderTM COVID-19 Plus RealAmp Kit assay (ELITechGroup, France). Moreover sperm examinations were performed according to WHO criteria.

Results: SARS-CoV-2 RNA has not been detected in semen samples from none of the subjects analysed. sperm analysis exhibited abnormal seminal parameters in 13 out of 15 patients (87\%). The inflammatory indices showed no differences in patients with abnormal semen compared to patients with normal semen. Conclusions: SARS-CoV-2 was not present in the semen while semen quality is altered in an high percentage of cases in this population. 